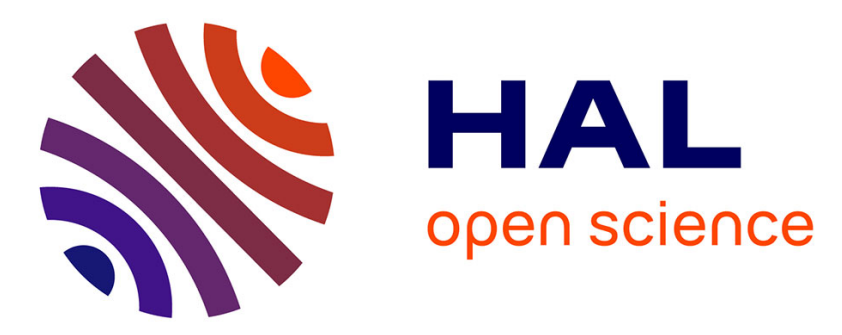

\title{
On the convergence of the rotated one-sided ergodic Hilbert transform
}

\author{
Nicolas Chevallier, Guy Cohen, Jean-Pierre Conze
}

\section{To cite this version:}

Nicolas Chevallier, Guy Cohen, Jean-Pierre Conze. On the convergence of the rotated one-sided ergodic Hilbert transform. Positivity, 2011, 15 (2), pp.253-270. 10.1007/s11117-010-0070-z . hal00456567

\section{HAL Id: hal-00456567 https://hal.science/hal-00456567}

Submitted on 15 Feb 2010

HAL is a multi-disciplinary open access archive for the deposit and dissemination of scientific research documents, whether they are published or not. The documents may come from teaching and research institutions in France or abroad, or from public or private research centers.
L'archive ouverte pluridisciplinaire HAL, est destinée au dépôt et à la diffusion de documents scientifiques de niveau recherche, publiés ou non, émanant des établissements d'enseignement et de recherche français ou étrangers, des laboratoires publics ou privés. 


\title{
ON THE CONVERGENCE OF THE ROTATED ONE-SIDED ERGODIC HILBERT TRANSFORM
}

\author{
NICOLAS CHEVALLIER, GUY COHEN, AND JEAN-PIERRE CONZE
}

\begin{abstract}
Sufficient conditions have been given for the convergence in norm and a.e. of the ergodic Hilbert transform $([11],[5],[6])$. Here we apply these conditions to the rotated ergodic Hilbert transform $\sum_{n=1}^{\infty} \frac{\lambda^{n}}{n} T^{n} f$, where $\lambda$ is a complex number of modulus 1. When $T$ is a contraction in a Hilbert space, we show that the logarithmic Hausdorff dimension of the set of $\lambda$ 's for which this series does not converge is at most 2 and give examples where this bound is attained.
\end{abstract}

\section{CONTEnTs}

\section{Introduction}

1. Ergodic Hilbert transform and rotated ergodic Hilbert transform 2

1.1. A lemma on Fourier series 2

1.2. The one-sided ergodic Hilbert transform 4

1.3. The rotated one-sided ergodic Hilbert transform $\sum_{n=1}^{\infty} \frac{\lambda^{n}}{n} T^{n} f \quad 6$

2. Examples 8

2.1. Moving averages 8

2.2. Examples with an uncountable set of $\lambda$ 's of non-convergence 10

3. Appendix: Hausdorff dimension of a set of divergence 12

3.1. h-Hausdorff dimension $\quad 12$

3.2. $h$-Hausdorff dimension of the set of divergence of the potential 13

References 16

\section{Introduction}

Let $T$ be a normal contraction on a Hilbert space $\mathcal{H}$. Let $D$ be the unit disk and, for $f \in \mathcal{H}$, denote the spectral measure of $f$ with respect to $T$ by $\sigma_{f}$. In [5] (see also [2] for earlier results) it was proved that the one-sided ergodic Hilbert transform (EHT) $\sum_{n=1}^{\infty} \frac{T^{n} f}{n}$ converges in $\mathcal{H}$ if and only if $\int_{D} \log ^{2}|1-z| d \sigma_{f}<\infty$. It is proved in [5] that when $T$ is a contraction (even not normal) on $\mathcal{H}=L_{2}(m)$ of a $\sigma$-finite measure, then

1991 Mathematics Subject Classification. Primary: 47A35, 47B15; Secondary: 37A30, 42A16.

Key words and phrases. contractions, one-sided rotated ergodic Hilbert transform. 
for $f \in L_{2}(m)$ the convergence of $\sum_{n=3}^{\infty} \frac{\left\langle T^{n} f, f\right\rangle \log n(\log \log \log n)^{2}}{n}$ insures $m$-a.e. and norm convergence of $\sum_{n=1}^{\infty} \frac{T^{n} f}{n}$. Convergence of the EHT is a strengthening of the convergence of the ergodic means given by the ergodic theorems.

Denote by $\Gamma$ the unit circle and take $\lambda \in \Gamma$. By the mean ergodic theorem we know that for every contraction $T$ the averages $\frac{1}{n} \sum_{k=1}^{n} \lambda^{k} T^{k} f$ converge in norm. When $T$ is induced by a measure-preserving transformation on a probability space $(\Omega, m)$, the Wiener-Wintner theorem [16] says that for $f \in L_{2}(\Omega, m)$ and for $m$-a.e. $\omega \in \Omega$, the averages $\frac{1}{n} \sum_{k=1}^{n} \lambda^{k} T^{k} f(\omega)$ converge for every $\lambda \in \Gamma$.

It is then natural to consider the convergence for $\lambda \in \Gamma$ of the rotated $\mathrm{EHT}$

$$
\sum_{n=1}^{\infty} \frac{\lambda^{n} T^{n} f}{n} .
$$

For the two-sided rotated ergodic Hilbert transform $\sum_{n=1}^{\infty} \frac{\lambda^{n} T^{n} f-\bar{\lambda}^{n} T^{* n} f}{n}$, for every contraction $T$ (not necessarily normal) on $\mathcal{H}$ and for every $f \in \mathcal{H}$, convergence in norm holds for every $\lambda \in \Gamma$ (Campbell [3]). Lacey and Terwilleger proved recently that if $T$ is induced by an invertible measure preserving transformation on a probability space, then for every $f \in L_{p}(m), p>1, m$-a.e. the two-sided ergodic Hilbert transform converges for every $\lambda \in \Gamma$ ([14], Corollary 7.2).

For the one-sided ergodic Hilbert transform, by Theorem 3.6 in [5] applied to $\lambda T$ and Carleson's theorem, convergence in norm holds for Lebesgue a.e. $\lambda$. The aim of this paper is to answer the following question: For a given $f \in \mathcal{H}$, what is the size of the set of $\lambda$ 's such that the series in (1) does not converge in norm ? We will show that the logarithmic Hausdorff dimension of this set is at most 2, and construct examples where it can be 2 . We consider also a.e. convergence when $\mathcal{H}$ is the space $L_{2}(m)$ of a $\sigma$-finite measure $m$.

Remark that, unlike the two-sided ergodic Hilbert transform, we cannot expect that outside a set of $m$-measure 0 , pointwise convergence holds for every $\lambda \in \Gamma$. Consider the map : $(x, y) \rightarrow(x, y+x)$ on the 2-torus. The spectrum is continuous on the subspace orthogonal to the functions depending only on $x$. For the function $f(x, y):=e^{2 \pi i y}$ there is convergence in norm, but the set of points $(x, y)$ such that pointwise convergence holds for every $\lambda$ is empty.

\section{Ergodic Hilbert transform and rotated ergodic Hilbert transform}

\subsection{A lemma on Fourier series.}

We begin with some preliminaries which slightly extend results of [5].

Let $b: u \rightarrow b(u)$ be a positive slowly varying function defined for $u \geq 1$ (i.e. for every $\delta>0, u^{\delta} b(u)$ is increasing and $u^{-\delta} b(u)$ is decreasing for $u$ large enough). Write $B(t):=\int_{1}^{t} \frac{b(u)}{u} d u$. 
It is known that, if $\sum_{n=1}^{\infty} \frac{b(n)}{n}=\infty$, then, as $t \rightarrow \infty, B(t) \cong \sum_{n \leq t} \frac{b(n)}{n}$ and $b(t)=o(B(t))$ (see [17], Ch. V, p. 188).

Lemma 1.1. Let $\nu$ be a finite measure on the interval $[-\pi, \pi[$. For a positive slowly varying function $b$ such that $\sum_{n=1}^{\infty} \frac{b(n)}{n}=\infty$, the following conditions are equivalent:

$$
\begin{aligned}
& \int_{-\pi}^{\pi} B\left(\frac{1}{|t|}\right) \nu(d t)<\infty ; \\
& \sum_{k=1}^{\infty} \int_{-\pi}^{\pi} \frac{e^{i k t} b(k)}{k} \nu(d t) \text { converges; } \\
& \liminf _{n \rightarrow+\infty} \sum_{k=1}^{n} \int_{-\pi}^{\pi} \frac{\cos (k t) b(k)}{k} \nu(d t)<+\infty .
\end{aligned}
$$

Proof. Clearly $(3) \Rightarrow(4)$. The proof of $(2) \Rightarrow(3)$ is similar to that of $(i) \Rightarrow(i i)$ of Theorem 3.3 given in [5] for the special case $b(k)=\log k$. We prove the general result for the sake of completeness.

Assume that (2) holds.

For every $n \geq 1$ and $t \in[-\pi, \pi] \backslash\{0\}$, we have $\left|\sum_{k=1}^{n} \mathrm{e}^{i k t}\right| \leq \frac{\pi}{2|t|}$. Since $b(u)$ is slowly varying, the sequence $\{b(n) / n\}_{n \geq 1}$ decreases to zero and Abel's summation by parts yields that the series $\sum_{n=1}^{\infty} \frac{\mathrm{e}^{i n t} b(n)}{n}$ converges for every $t \in[-\pi, \pi] \backslash\{0\}$, and that the partial sums are uniformly bounded on the set $\{t \in[-\pi, \pi]:|t| \geq \varepsilon>0\}$ for every $\varepsilon>0$.

As $\nu(\{0\})=0$ by $(2), \sum_{k=1}^{\infty} \frac{\mathrm{e}^{i k t} b(k)}{k}$ converges $\nu$-a.e. To prove (3), by the Lebesgue dominated convergence theorem and by (2), it suffices to prove that, for $t$ in a neighborhood of $0, \sup _{n \geq 1}\left|\sum_{k=1}^{n} \frac{\mathrm{e}^{i k t} b(k)}{k}\right|$ is dominated by $B\left(|t|^{-1}\right)$.

We will bound $\sup _{n \geq 1}\left|\sum_{k=1}^{n} \frac{\mathrm{e}^{i k t} b(k)}{k}\right|$ for $0<|t| \leq \frac{1}{3}$. Let $n_{t}:=\left[|t|^{-1}\right]$.

For $n \leq n_{t}$, we have:

$$
\left|\sum_{k=1}^{n} \frac{\mathrm{e}^{i k t} b(k)}{k}\right| \leq \sum_{k=1}^{n} \frac{b(k)}{k} \leq C B(n) \leq C B\left(|t|^{-1}\right) .
$$

For $n>n_{t}$, we use the decomposition

$$
\sum_{k=1}^{n} \frac{\mathrm{e}^{i k t} b(k)}{k}=\sum_{k=1}^{n_{t}} \frac{\mathrm{e}^{i k t} b(k)}{k}+\sum_{k=n_{t}+1}^{n} \frac{\mathrm{e}^{i k t} b(k)}{k}=P_{1}+P_{2}
$$


with $P_{1}$ estimated above. Let $S_{j}:=\sum_{k=1}^{j} \mathrm{e}^{i k t}$, for $j \geq 1$. Since $n \geq n_{t}+1>|t|^{-1}$ and $\{b(n) / n\}$ is decreasing, using Abel's summation, we obtain

$$
\begin{aligned}
\left|P_{2}\right| & \leq \frac{b(n)}{n}\left|S_{n}\right|+\sum_{k=n_{t}+1}^{n-1}\left(\frac{b(k)}{k}-\frac{b(k+1)}{k+1}\right)\left|S_{k}\right|+\frac{b\left(n_{t}+1\right)}{n_{t}+1}\left|S_{n_{t}}\right| \\
& \leq \frac{b(n)}{n} \frac{\pi}{2|t|}+2 \frac{b\left(n_{t}+1\right)}{n_{t}+1} \frac{\pi}{2|t|} \leq \frac{3 \pi}{2} \frac{b\left(|t|^{-1}\right)}{|t|^{-1}} \frac{1}{|t|}=\frac{3 \pi}{2} b\left(|t|^{-1}\right) .
\end{aligned}
$$

The two cases together give

$$
\sup _{n \geq 1}\left|\sum_{k=1}^{n} \frac{\mathrm{e}^{i k t} b(k)}{k}\right| \leq C^{\prime} b\left(|t|^{-1}\right)+C B\left(|t|^{-1}\right), \forall t \in[-\pi, \pi] \backslash\{0\} .
$$

This prove our claim, since $b(u)=o(B(u))$ as $u \rightarrow \infty$.

Now we prove $(4) \Rightarrow(2)$. For $\alpha \in] 0,1\left[\right.$, the partial sums $\sum_{k=1}^{n} \frac{\cos (k t)}{k^{\alpha}}$ are uniformly bounded from below (see Zygmund ([17], Ch. V, Th. 2.29)). Hence, by Abel's summation by parts (using the fact that $\left\{b(n) / n^{1-\alpha}\right\}$ decreases) the partial sums $\sum_{k=1}^{n} \frac{\cos (k t) b(k)}{k}$ are uniformly bounded from below, say by $-C$.

We have $\nu(\{0\})=0$, since (4) implies

$$
0 \leq \liminf _{n \rightarrow \infty} \int_{-\pi}^{\pi}\left(C+\sum_{k=1}^{n} \frac{\cos (k t) b(k)}{k}\right) \nu(d t)<\infty .
$$

Using again that the sequence $\{b(k) / k\}$ decreases to zero, and Abel's summation by parts, we have the convergence of the series $\sum_{k=1}^{\infty} \frac{\cos (k t) b(k)}{k}$ for every $0 \neq t \in[-\pi, \pi]$, hence its convergence $\nu$-a.e., and by Fatou's lemma

$$
\int_{-\pi}^{\pi} \lim _{n \rightarrow \infty}\left(C+\sum_{k=1}^{n} \frac{\cos (k t) b(k)}{k}\right) \nu(d t) \leq \liminf _{n \rightarrow \infty} \int_{-\pi}^{\pi}\left(C+\sum_{k=1}^{n} \frac{\cos (k t) b(k)}{k}\right) \nu(d t) .
$$

The integrand in (2) is bounded for $|t| \geq \varepsilon>0$. Since $\sum_{k=1}^{\infty} \frac{\cos (k t) b(k)}{k}$ behaves like $B\left(|t|^{-1}\right)$ as $t \rightarrow 0$ (see Zygmund ([17], Ch. V, Th. 2.15)), condition (2) is satisfied.

Note that although we only assume that the liminf in (4) is not $+\infty$, the proof shows that it can not be $-\infty$, and that in fact the series converges.

\subsection{The one-sided ergodic Hilbert transform.}

Let $T$ be a contraction of a Hilbert space $\mathcal{H}$. Define $T_{n}:=T^{n}$ for $n \geq 0$ and $T_{n}:=\left(T^{*}\right)^{|n|}$ for $n<0$. Then $\left\{\left\langle T_{n} f, f\right\rangle\right\}$ is a positive semi-definite sequence ([15], Appendix, §9) and therefore by Herglotz's theorem it is the sequence of the Fourier coefficients of a positive finite measure $\nu_{f}$ on the unit circle $\Gamma$. We will still denote by $\nu_{f}$ the representation of the measure $\nu_{f}$ as a measure on the interval $I=[-\pi, \pi[$ and use both representations. 
By the unitary dilation theorem of B. Sz. Nagy ([15], Theorem III, p. 469), there exist a larger Hilbert space $\mathcal{H}^{\prime}$, an orthogonal projection $P_{\mathcal{H}}$ from $\mathcal{H}^{\prime}$ onto $\mathcal{H}$, and an unitary operator $U$ on $\mathcal{H}^{\prime}$ such that

$$
T_{n} P_{\mathcal{H}} g=P_{\mathcal{H}} U^{n} g, \forall g \in \mathcal{H}^{\prime}, \forall n \in \mathbb{Z}
$$

For $f \in \mathcal{H}$, the above identity yields

$$
\left\langle T_{n} f, f\right\rangle=\left\langle P_{\mathcal{H}} U^{n} f, f\right\rangle=\left\langle U^{n} f, P_{\mathcal{H}}^{*} f\right\rangle=\left\langle U^{n} f, P_{\mathcal{H}} f\right\rangle=\left\langle U^{n} f, f\right\rangle .
$$

By the spectral representation theorem for unitary operators, $\nu_{f}$ is the spectral measure of $f$ with respect to $U$, with Fourier coefficients $\left\{\hat{\nu}_{n}=\left\langle T_{n} f, f\right\rangle\right\}$.

Definition 1.2. For a contraction $T$ on $\mathcal{H}$ and $f \in \mathcal{H}, \nu_{f}$ is called the unitary spectral measure of $f$ (with respect to $T$ ). When $\nu_{f}$ is absolutely continuous with respect to the Lebesgue measure, we say that $f$ has a spectral density.

Let $b(u)$ be a positive slowly varying function such that $\sum_{n=1}^{\infty} \frac{b(n)}{n}=\infty$. With the previous notations, the equivalence given by Lemma 1.1 yields immediately the equivalence between the following conditions:

$$
\begin{aligned}
& \int_{-\pi}^{\pi} B\left(\frac{1}{|t|}\right) \nu_{f}(d t)<\infty \\
& \sum_{k=1}^{\infty} \int_{-\pi}^{\pi} \frac{\mathrm{e}^{i k t} b(k)}{k} \nu_{f}(d t) \text { converges. }
\end{aligned}
$$

If $T$ is a normal contraction, then the previous conditions are equivalent to

$$
\sum_{n=1}^{\infty} \frac{\left\|\sum_{k=1}^{n} T^{k} f\right\|^{2} b(n)}{n^{3}}<\infty
$$

Indeed the proof of the equivalence between (5), (6) and (7) for the special case $b(n)=$ $\log n$ (and hence $B(u)=\log ^{2} u$ ) was given in [5], Theorem 3.3, for $T$ a normal contraction and can be adapted for a more general $b(n)$.

Let us also mention Cuny ([7], Lemma 2.1) for the equivalence (5) $\Leftrightarrow(7)$ and, for the case $b(n)=\log n(\log \log \log n)^{2}$, Gaposhkin ([11], conditions (33) and (34)) who has indicated that in the unitary case, with this choice of $b(n),(5)$ and (7) are equivalent and both are implied by (6). Here we see that these three conditions are equivalent.

Theorem 1.3. 1) Let $T$ be a contraction on a Hilbert space $\mathcal{H}$ and $f \in \mathcal{H}$ with unitary spectral measure $\nu_{f}$. Then the following conditions are equivalent:

$$
\begin{aligned}
& \int_{-\pi}^{\pi} \log ^{2}|t| \nu_{f}(d t)<\infty, \\
& \sum_{n=1}^{\infty} \frac{\left\langle T^{n} f, f\right\rangle \log n}{n} \text { converges. }
\end{aligned}
$$


They imply

$$
\sum_{n=1}^{\infty} \frac{T^{n} f}{n} \text { converges in norm. }
$$

2) If $T$ is a normal contraction, then (8), (9), (10) and (11) below (where $\sigma_{f}$ is the spectral measure of $f$ ) are equivalent

$$
\int_{D} \log ^{2}|1-z| \sigma_{f}(d z)<\infty
$$

3) If $T$ is a contraction of $L_{2}(m)$ of a $\sigma$-finite measure space and $f$ is in $L_{2}(m)$, the convergence of the series $\sum_{n=1}^{\infty} \frac{\left\langle T^{n} f, f\right\rangle \log n(\log \log \log n)^{2}}{n}$ implies the convergence in norm and $m$-a.e. of $\sum_{n=1}^{\infty} \frac{T^{n} f}{n}$.

Proof. The theorem is essentially in [5], except that it is shown here that all the information about the convergence of the one-sided EHT is contained in the unitary spectral measure, since the equivalence $(8) \Leftrightarrow(9)$ is a particular case of $(5) \Leftrightarrow(6)$.

The implication $(9) \Rightarrow(10)$ is Theorem 4.2 in [5], where also the equivalence $(11) \Leftrightarrow$ $(9) \Leftrightarrow(10)$ is shown for a normal contraction. For the a.e. and norm convergence, see Theorem 4.3 in [5].

Remarks 1) If $T$ is a normal contraction and $U$ its unitary dilation, then $\sum_{n=1}^{\infty} \frac{U^{n} f}{n}$ converges if and only if $\sum_{n=1}^{\infty} \frac{T^{n} f}{n}$ converges. Indeed, the "only if" follows by the continuity of the projection $P_{\mathcal{H}}$. For the "if" condition we apply the theorem to the unitary operator $U$, since (9) holds for $U$.

2) As mentioned in [5], if $T$ is an isometry and $U$ its unitary dilation, then $T^{n} f=U^{n} f$ for every $f \in \mathcal{H}$. Hence, when $T$ is an isometry, $(8) \Leftrightarrow(9) \Leftrightarrow(10)$.

3) Gaposhkin [11] has shown that in the family of all unitary operators $T$, the condition $\int_{-\pi}^{\pi} b(t) \nu_{f}(d t)<\infty$ with $b(t):=\left(\log \left(|t|^{-1}\right) \log \log \left|\log \left(|t|^{-1}\right)\right|\right)^{2}$ is sharp. As well, by the equivalence $(2) \Leftrightarrow(3)$ in Lemma 1.1, the factor $\log n(\log \log \log n)^{2}$ in claim 3) of Theorem 1.3 can not be replaced by any slowly varying function $b(u)$ with $\int_{1}^{\infty} \frac{b(u)}{u} d u=\infty$ and $b(n)=o\left(\log n(\log \log \log n)^{2}\right)$ for the class of unitary operators.

\subsection{The rotated one-sided ergodic Hilbert transform $\sum_{n=1}^{\infty} \frac{\lambda^{n}}{n} T^{n} f$.}

Let $T$ be a contraction on $\mathcal{H}$ and let $f \in \mathcal{H}$. Let $\nu_{f}$ be the unitary spectral measure of $f$ with respect to $T$. For $\lambda \in \Gamma$ we have $(\lambda T)_{n}=\lambda^{n} T_{n}$, for every $n \in \mathbb{Z}$. Hence the unitary spectral measure of $f$ with respect to $\lambda T$ is $\nu_{f}\left(\lambda^{-1}\right.$.) (denoted by $\left.\delta_{\lambda} * \nu_{f}\right)$.

Similarly, if $T$ is a normal contraction on $\mathcal{H}$ and if $\sigma_{f}$ is the spectral measure of $f$ with respect to $T$, then the spectral measure of $f$ with respect to $\lambda T$ is $\sigma_{f}\left(\lambda^{-1} \cdot\right)$. Therefore the following proposition results immediately from Theorem 1.3. 
Proposition 1.4. Let $T$ be a contraction on a Hilbert space $\mathcal{H}$, let $f$ be in $\mathcal{H}$ with unitary spectral measure $\nu_{f}$, and let $\lambda \in \Gamma$.

1) Then the following conditions are equivalent:

$$
\begin{aligned}
& \int_{\Gamma} \log ^{2}|1-z| \delta_{\lambda} * \nu_{f}(d z)<\infty \\
& \sum_{n=1}^{\infty} \frac{\lambda^{n}\left\langle T^{n} f, f\right\rangle \log n}{n} \text { converges. }
\end{aligned}
$$

They imply

$$
\sum_{n=1}^{\infty} \frac{\lambda^{n} T^{n} f}{n} \text { converges in norm. }
$$

2) If $T$ is a normal contraction, then (12), (13), (14) and (15) below are equivalent

$$
\int_{D} \log ^{2}|1-z| \delta_{\lambda} * \sigma_{f}(d z)<\infty
$$

3) Assume that $T$ is contraction of the space $L_{2}(m)$ of a $\sigma$-finite measure space and $f \in L_{2}(m)$.

Then the convergence of the series $\sum_{n=1}^{\infty} \frac{\lambda^{n}\left\langle T^{n} f, f\right\rangle \log n(\log \log \log n)^{2}}{n}$ implies that

$$
\sum_{n=1}^{\infty} \frac{\lambda^{n} T^{n} f}{n} \text { converges in norm and } m-\text { a.e. }
$$

Remarks 1) If $T$ be a positive contraction on $L_{2}(m)$ of a $\sigma$-finite measure $m$, or if $T$ is a Dunford-Schwartz operator, then $\sum_{n=1}^{\infty} \frac{\lambda^{n} T^{n} f}{n}$ converges $m$-a.e. for $\lambda \in \Gamma$ such that (14) holds. Since the modulus of $\lambda T$ is $T$ in the first case, the linear modulus of $T$ in the second case, this results from Theorem 2.1 in Cuny [6] applied to $\lambda T$.

2) When the measure $\nu_{f}$ is absolutely continuous and $d \nu_{f} / d t \in L_{p}(d t)$, for some $p>1$, then, as $(\log (1 /|t|))^{2}(\log \log |\log (1 /|t|)|)^{2} \in L_{q}([-\pi, \pi[, d t)$ for every $1 \leq q<\infty$, Hölder's inequality implies that (16) holds for every $\lambda \in \Gamma$.

$3)$ If $T$ is induced by an ergodic dynamical system defined on a probability space $(\Omega, m)$ and if $T$ has Lebesgue spectrum, then there is a dense set of functions in the space $L_{2}^{0}(m)$ of functions in $L_{2}(m)$ with zero integral such that (16) holds for every $\lambda \in \Gamma$.

Indeed, when $T$ has Lebesgue spectrum, there is an orthogonal decomposition $\bigoplus_{j \in J} H_{j}$ of the space $L_{2}^{0}(m)$, where $J$ is the spectral multiplicity, and $H_{j}, j \in J$, is the closed subspace of $L_{2}^{0}(m)$ spanned by $\left\{T^{k} f_{j}, k \in \mathbb{Z}\right\}$ for some function $f_{j} \in L_{2}^{0}(m)$ such that $\left\langle f_{j}, T^{k} f_{j}\right\rangle=0$, for every $k \neq 0$. The finite linear combinations of $\left\{T^{k} f_{j}, j \in J, k \in \mathbb{Z}\right\}$ are dense in $L_{2}^{0}(m)$ and these functions have a polynomial spectral density. The result then follows from Remark 2). 
4) If (14) holds for some $\lambda$, then it holds for the orthogonal projection of $f$ on any $T$ invariant subspace, hence $f$ is orthogonal to the eigenspace corresponding to the eigenvalue $\bar{\lambda}$ (if there is a $\bar{\lambda}$-eigenfunction) and $\sigma_{f}(\{\bar{\lambda}\})=0$.

Proposition 1.4 shows that, for a normal contraction, if we have norm convergence of $\sum_{n=1}^{\infty} \frac{\lambda^{n} T^{n} f}{n}$ for $f \in \mathcal{H}$ and for every $\lambda \in \Gamma$, then it is not only that $\sigma_{f}$ is a continuous measure but it has a rate in its modulus of continuity. For every subset $B \subset D$ containing $\bar{\lambda}$, with $0<\delta=\sup _{z \in B}|z-\bar{\lambda}| \leq 1$, we have

$$
\sigma_{f}(B)=\int_{B} d \sigma_{f} \leq \frac{1}{\log ^{2} \delta} \int_{D} \log ^{2}|1-\lambda z| d \sigma_{f}(z) \leq \frac{C_{\lambda}}{\log ^{2} \delta} .
$$

5) For any aperiodic dynamical system and any $\lambda \in \Gamma$, there is a dense $G_{\delta}$ set of functions $f \in L_{2}^{0}(m)$ such that (16) does not hold (del Junco and Rosenblatt, see Remark following Corollary 3.3 in [12]).

We construct in Section 2 a stationary process such that the set of $\lambda$ 's for which (16) does not hold is "big" in some sense. The same construction can be performed for any dynamical system with Lebesgue spectrum and provides functions $f$ such that the set of $\lambda \in \Gamma$ for which (16) does not hold has a logarithmic Hausdorff dimension 2.

In the opposite direction, for every contraction, 2 is always a bound for logarithmic Hausdorff dimension of the set of such $\lambda$ 's:

Theorem 1.5. Let $T$ be a contraction on a Hilbert space $\mathcal{H}$ and $f \in \mathcal{H}$. The set of $\lambda \in \Gamma$ such that $\sum_{n=1}^{\infty} \frac{\lambda^{n} T^{n} f}{n}$ does not converge in norm has a logarithmic Hausdorff dimension at most 2.

Proof. By Proposition 1.4 the set of non-convergence is included in the set $\left\{\lambda: \int_{\Gamma} \log ^{2} \mid 1-\right.$ $\left.z \mid \delta_{\lambda} * \nu_{f}(d z)=+\infty\right\}$. The result then follows from Theorem 3.2 on $h$-Hausdorff dimensions (see Appendix), with $h$ defined by $h(0)=0, h(x)=1 /|\log x|$ for $0<x \leq 1 / 2$, and $h(x)=1 / \log 2$, for $x>1 / 2$.

\section{Examples}

\subsection{Moving averages.}

Let $\left\{\xi_{k}\right\}$ be a sequence of centered i.i.d. complex random variables on a probability space $(\Omega, m)$ with $\mathbb{E}\left|\xi_{1}\right|^{2} d m=1$. Let $\left\{c_{k}\right\}_{k \in \mathbb{Z}}$ be a sequence in $\ell_{2}(\mathbb{Z})$ and $c(t):=\sum_{k=-\infty}^{\infty} c_{k} \mathrm{e}^{i k t}$.

For $n \in \mathbb{Z}$, we define the moving averages $f_{n}:=\sum_{k=-\infty}^{\infty} c_{k} \xi_{n+k}$. This series converges in $L_{2}(m)$ by the Riesz-Fischer theorem, and almost everywhere by the KhintchineKolmogorov theorem. Clearly $f_{n}=T^{n} f_{0}$, where $T$ is induced by the two-sided shift that generates $\left\{\xi_{k}\right\}$. The spectral measure $\nu$ of $f_{0}$ with respect to $T$ is absolutely continuous and $\frac{d \nu}{d t}(t)=|c(t)|^{2}$. Conversely, for any function $c \in L_{2}\left(\left[-\pi, \pi[, d t),|c(t)|^{2}\right.\right.$ is the spectral density of a moving average. Therefore, for any nonnegative function $g$ with

$\int_{-\pi}^{\pi} g d t=1$, there is a stationary moving average model with $g$ as spectral density. If we choose $\left\{\xi_{k}\right\}$ to be Gaussian, then the resulting stationary process is also Gaussian. 
In terms of the function $c$ which generates the moving averages, the condition for the convergence (condition (12) of Proposition 1.4) reads, for $\lambda=e^{i s}, \int_{-\pi}^{\pi}|c(t)|^{2} \log ^{2} \mid s-$ $t \mid d t<\infty$. Since $T$ in the examples of this section is unitary, (12) is equivalent to the convergence in norm of the rotated EHT.

The next proposition shows that, for this class of examples, the set of $\lambda$ 's where $\sum_{n=1}^{\infty} \frac{\lambda^{n} f_{n}}{n}$ does not converge is the same for the convergence in probability, in norm, and a.e. That is, in general, we can not reduce the size of this set by weakening the mode of convergence.

Proposition 2.1. Assume that the random variables $\left\{\xi_{k}\right\}$ are in $L_{4}(\Omega, m)$. Then, for any sequence of complex numbers $\left\{a_{n}\right\}$, the convergence in probability and the convergence in $L_{2}$-norm of $\sum_{n=1}^{\infty} a_{n} f_{n}$ are equivalent.

When $a_{n}=\lambda^{n} / n$, convergence in probability, convergence in norm, and a.e. convergence are equivalent.

Proof. For $k \in \mathbb{Z}$ and $N \geq 1$, let $b_{k}^{N}:=\sum_{n=1}^{N} a_{n} c_{k-n}$. Since $\left\{c_{k}\right\}$ is in $\ell_{2}(\mathbb{Z})$, for every $N \geq 1$ we have $b^{N}:=\left\{b_{k}^{N}\right\}_{k \in \mathbb{Z}} \in \ell_{2}(\mathbb{Z})$, and

$$
\sum_{n=1}^{N} a_{n} f_{n}=\sum_{k=-\infty}^{\infty} \xi_{k} \sum_{n=1}^{N} a_{n} c_{k-n}=\sum_{k=-\infty}^{\infty} \xi_{k} b_{k}^{N}
$$

Assume convergence in probability of the sequence $\left(\sum_{n=1}^{N} a_{n} f_{n}\right)_{N \geq 1}$. By (17) we conclude that for every $\varepsilon>0$,

$$
\lim _{N, M \rightarrow \infty} m\left(\left|\sum_{k=-\infty}^{\infty} \xi_{k}\left(b_{k}^{N}-b_{k}^{M}\right)\right|>\varepsilon\right)=0 .
$$

Now we prove that $\left\{b^{N}\right\}_{N \geq 1}$ is a Cauchy sequence in $\ell_{2}(\mathbb{Z})$. Otherwise, there would exist $\varepsilon_{0}>0$ and a sequence of integers $N_{j} \uparrow \infty$, such that $\left\|b^{N_{j+1}}-b^{N_{j}}\right\|_{2} \geq \varepsilon_{0}$. Since $\xi_{k} \in L_{4}$, by the Paley-Zygmund inequality (cf. [13], p. 31, Theorem 3), for a fixed $\kappa \in] 0,1$ [ there exists $\eta>0$, such that

$$
\begin{aligned}
& m\left(\left|\sum_{k=-\infty}^{\infty} \xi_{k}\left(b_{k}^{N_{j+1}}-b_{k}^{N_{j}}\right)\right|>\kappa \varepsilon_{0}\left\|\xi_{0}\right\|_{2}\right) \geq \\
& m\left(\left|\sum_{k=-\infty}^{\infty} \xi_{k}\left(b_{k}^{N_{j+1}}-b_{k}^{N_{j}}\right)\right|>\kappa\left\|\xi_{0}\right\|_{2}\left\|b^{N_{j+1}}-b^{N_{j}}\right\|_{2}\right)>\eta .
\end{aligned}
$$

Since the left hand side of the above inequality tends to zero as $j \rightarrow \infty$, we have a contradiction.

Hence $\left\{b^{N}\right\}_{N \geq 1}$ is a Cauchy sequence and therefore converges to some sequence $b=$ $\left\{b_{k}\right\} \in \ell_{2}(\mathbb{Z})$. Using $(17)$ we obtain

$$
\left\|\sum_{n=N+1}^{M} a_{n} f_{n}\right\|^{2}=\left\|\sum_{k=-\infty}^{\infty} \xi_{k}\left(b_{k}^{M}-b_{k}^{N}\right)\right\|^{2}=\left\|b^{M}-b^{N}\right\|^{2}\left\|\xi_{0}\right\|^{2} \longrightarrow 0 .
$$

This implies that $\sum_{n=1}^{N} a_{n} f_{n}$ converges in norm to $\sum_{k=-\infty}^{\infty} b_{k} \xi_{k}$, with $b_{k}=\sum_{n=1}^{\infty} a_{n} c_{k-n}$. 
When $a_{n}=\lambda^{n} / n$, the equivalence with a.e. convergence follows from the result of Cuny [6, Theorem 2.1] mentioned in the remarks of Section 1.3.

\subsection{Examples with an uncountable set of $\lambda$ 's of non-convergence.}

We construct now by a different method stationary Gaussian processes, first with a countable set of non-convergence, then with an uncountable set.

Proposition 2.2. There is a Gaussian stationary process $\left\{X_{n}\right\}$ with a spectral density such that the series $\sum_{n=1}^{\infty} \frac{\lambda^{n} X_{n}}{n}$ does not converge in norm for $\lambda$ in an infinite countable subset of $\Gamma$.

Proof. The computations are done on the interval $\left[-\pi, \pi\left[\right.\right.$. Let $\left\{s_{k}\right\}$ be a sequence in $\left[0, \mathrm{e}^{-1}\right)$ and let $\left\{c_{k}\right\} \in \ell_{1}$ be a positive sequence. On $[-\pi, \pi]$, define $g_{k}(t):=$

$\frac{\mathbf{1}_{\left[s_{k}, \mathrm{e}^{-1}\right]}(t)}{\left(t-s_{k}\right) \log ^{2}\left(t-s_{k}\right)}$. Since the integral on $[-\pi, \pi]$ of $g_{k}$ is less than $\int_{0}^{\mathrm{e}^{-1}} \frac{d t}{t \log ^{2}(t)}=1$, the series $g(t):=\sum_{k=1}^{\infty} c_{k} g_{k}(t)$ is a.e. convergent and defines an integrable function $g$ such that $\|g\|_{1} \leq \sum_{k=1}^{\infty} c_{k}$.

By Doob [8, Th. 3.1, p. 72], there is a stationary Gaussian process $\left\{X_{n}\right\}$ with spectral density $g$. Let $T$ be the transformation such that $X_{n}=T^{n} X_{0}$. The spectral density of $X_{0}$ with respect to $T$ is $g$. As the series is positive:

$$
\int_{-\pi}^{\pi} \log ^{2}|t-s| g(t) d t=\sum_{k=1}^{\infty} \int_{s_{k}}^{\mathrm{e}^{-1}} \log ^{2}|t-s| \frac{c_{k}}{\left(t-s_{k}\right) \log ^{2}\left(t-s_{k}\right)} d t .
$$

Hence, the integral on the left hand side is infinite for every $s \in\left\{s_{k}\right\}$.

Now we modify this example to get a set of nonconvergence of positive logarithmic Hausdorff dimension. (See Assani [1] for a related question).

Proposition 2.3. For every $\alpha<2$, there exists a non-empty perfect nowhere dense subset $P \subset \Gamma$, with logarithmic Hausdorff dimension $\geq \alpha$, and a Gaussian stationary process $\left\{X_{n}\right\}$, with a spectral density, such that the series $\sum_{n=1}^{\infty} \frac{\lambda^{n} X_{n}}{n}$ does not converge in norm for every $\lambda \in P$.

Proof. The construction of $\left\{X_{n}\right\}$ is like in the previous example, but we change the definition of $\left\{s_{k}\right\}$ and $g_{k}$. We build a sequence $\left\{s_{k}\right\} \subset\left[0, \mathrm{e}^{-\mathrm{e}}\right]$ whose elements are the endpoints of intervals in the construction of a Cantor type set of a non-constant ratio of dissection. The closure of $\left\{s_{k}\right\}$ will be a uncountable perfect nowhere dense set. Observe that each of the end points appears infinitely often in the sequence $\left\{s_{k}\right\}$.

For any interval $[x, x+l]$ and $\eta \in] 0,1 / 2[$, let us consider the closed disjoint intervals $[x, x+l \eta]$ and $[x+l(1-\eta), x+l]$. These intervals are called 'end intervals' and the complementary open interval with respect to $[x, x+l]$, called 'middle interval', is removed. This dissection of $[x, x+l]$ will be said of type $[2 ; \eta]$. Let $\varepsilon>0, \eta_{1}:=1 / 3^{(2+\varepsilon)^{\frac{1}{2}}}$, and

$$
\eta_{k}=\left[\eta_{k-1} \cdot \eta_{k-2} \cdots \eta_{1}\right]^{-1} 3^{-(2+\varepsilon)^{\frac{k}{2}}}=\frac{3^{(2+\varepsilon)^{\frac{k-1}{2}}}}{3^{(2+\varepsilon)^{\frac{k}{2}}}}, k \geq 2 .
$$


Starting from $\left[0, \mathrm{e}^{-\mathrm{e}}\right]$, we perform a dissection of type $\left[2 ; \eta_{1}\right]$ and remove the middle interval. On each remaining end interval we perform a dissection of type $\left[2 ; \eta_{2}\right]$, and remove the middle intervals - and so on. After $n$ operations we have $2^{n}$ end intervals, each of length $\mathrm{e}^{-\mathrm{e}} \eta_{n} \eta_{n-1} \cdots \eta_{1}$. As $n \rightarrow \infty$ we obtain a non-empty perfect nowhere dense set $P$ (necessarily uncountable) of Lebesgue measure zero.

The sequence $\left\{s_{k}\right\}$ is defined as follows. At the first dissection we have two end intervals $\left[s_{1}, s_{2}\right]$ and $\left[s_{3}, s_{4}\right]$. At the second dissection we have 4 end intervals. Let $s_{5}, \ldots, s_{12}$ be their endpoints (in increasing order) - and so on. One easily sees that the closure of $\left\{s_{k}\right\}$ is the same Cantor type set $P$ obtained in the dissection process above.

Let $B_{n}$ be the set of indexes $k$ such that $s_{k}$ belongs to the $n$-th operation. Let $c_{k}:=\frac{1}{(2+\varepsilon)^{n}}$, for $k \in B_{n}$. Since $B_{n}$ contains $2^{n+1}$ elements, clearly $\left\{c_{k}\right\} \in \ell_{1}$.

The functions $g_{k}$ are defined by:

$$
g_{k}(t):=\frac{\mathbf{1}_{\left[s_{k}, \mathrm{e}^{-\mathrm{e}]}\right.}(t)}{\left(t-s_{k}\right)\left|\log \left(t-s_{k}\right)\right|\left(\log \left|\log \left(t-s_{k}\right)\right|\right)^{2}} .
$$

The function $g(t):=\sum_{k=1}^{\infty} c_{k} g_{k}(t)$ is integrable with $\|g\|_{1}=\sum_{k=1}^{\infty} c_{k} \int_{-\pi}^{\pi} g_{k}(t) d t \leq$ $\sum_{k=1}^{\infty} c_{k}$, and we have the following lower bound for every $s \in\left[0, \mathrm{e}^{-\mathrm{e}}\right]$ :

$$
\begin{aligned}
& \int_{-\pi}^{\pi} \log ^{2}|t-s| g(t) d t \geq \sum_{k: s_{k}<s} c_{k} \int_{s_{k}}^{\frac{1}{2}\left(s+s_{k}\right)} \frac{\log ^{2}|t-s| d t}{\left(t-s_{k}\right)\left|\log \left(t-s_{k}\right)\right|\left(\log \left|\log \left(t-s_{k}\right)\right|\right)^{2}} \\
& \geq \sum_{k: s_{k}<s} c_{k} \log ^{2}\left(s-s_{k}\right) \int_{s_{k}}^{\frac{1}{2}\left(s+s_{k}\right)} \frac{d t}{\left(t-s_{k}\right)\left|\log \left(t-s_{k}\right)\right|\left(\log \left|\log \left(t-s_{k}\right)\right|\right)^{2}} \\
& =\sum_{k: s_{k}<s} c_{k} \frac{\log ^{2}\left(s-s_{k}\right)}{|\log | \log \left(\frac{1}{2}\left(s-s_{k}\right)\right)||} .
\end{aligned}
$$

From the construction it follows that, for any $s \in P$ and any integer $n \geq 1$, there exists an interval endpoint $s(n)$, such that $s(n)<s$ with $s-s(n) \leq \mathrm{e}^{-\mathrm{e}} \eta_{n} \cdots \eta_{1}<3^{-(2+\varepsilon)^{\frac{n}{2}}}$, and $s(n)$ belongs to the $n$-th operation. Hence, for $s \in P$ :

$$
\begin{aligned}
& \int_{-\pi}^{\pi} \log ^{2}|t-s| g(t) d t \geq \sum_{k: s_{k}<s} c_{k} \frac{\log ^{2}\left(s-s_{k}\right)}{|\log | \log \left(\frac{1}{2}\left(s-s_{k}\right)\right)||} \\
& =\sum_{n=1}^{\infty} \sum_{k \in B_{n}, s_{k}<s} c_{k} \frac{\log ^{2}\left(s-s_{k}\right)}{|\log | \log \left(\frac{1}{2}\left(s-s_{k}\right)\right)||} \geq \sum_{n=1}^{\infty} \frac{1}{(2+\varepsilon)^{n}} \frac{\log ^{2}(s-s(n))}{|\log | \log \left(\frac{1}{2}(s-s(n))\right) \mid} \\
& \geq \sum_{n=1}^{\infty} \frac{1}{(2+\varepsilon)^{n}} \frac{\left[(2+\varepsilon)^{\frac{n}{2}} \log 3\right]^{2}}{\left|\log \left[(2+\varepsilon)^{\frac{n}{2}} \log 3-\log 2\right]\right|}=+\infty
\end{aligned}
$$

Define the function $K(x)=\left(\log (1 / x)^{+}\right)^{\alpha}, \alpha>0$. Using Carleson [4, §IV, Theorem 3], we conclude that $P$ has a positive $K$-capacity if and only if the series $\sum_{k=1}^{\infty} 2^{-k} K\left(\eta_{k}\right)$ 
converges. According to (18), we have $\sum_{k=2}^{\infty} 2^{-k} K\left(\eta_{k}\right)=\log ^{\alpha}(3) \sum_{k=2}^{\infty} 2^{-k}\left[(2+\varepsilon)^{\frac{k}{2}}-\right.$ $\left.(2+\varepsilon)^{\frac{k-1}{2}}\right]^{\alpha}$ and the series converges if and only if $\alpha<2 \log (2) / \log (2+\varepsilon)$. Theorem 1 in $[4, \S I V]$ implies that the logarithmic Hausdorff dimension is $\geq \alpha$. As $\varepsilon$ is arbitrary the assertion follows.

The bound 2 of the logarithmic Hausdorff dimension of the set of $\lambda \in \Gamma$ such that $\sum_{n=1}^{\infty} \frac{\lambda^{n} T^{n} f}{n}$ does not converge in norm can be attained:

Theorem 2.4. There exist an uncountable subset $P \subset \Gamma$ with logarithmic Hausdorff dimension 2 and a Gaussian stationary process $\left\{X_{n}\right\}$ with a spectral density, such that the series $\sum_{n=1}^{\infty} \frac{\lambda^{n} X_{n}}{n}$ does not converge in norm for every $\lambda \in P$. The closure of $P$ is a perfect set.

Proof. Take $\alpha_{j} \uparrow 2$ and for every $j \geq 1$ build the associated function $g_{j}$ and and set $P_{j}$ as in Proposition 2.3. We can assume that $\left\|g_{j}\right\|_{1}=1$ for every $j \geq 1$. Now, define $P=$ $\cup_{j=1}^{\infty} P_{j}$ and put $g=\sum_{j=1}^{\infty} \beta_{j} g_{j}$, where $\left\{\beta_{j}\right\}$ is a summable sequence of positive numbers. Clearly, $g$ is an integrable function and there is a Gaussian stationary process $\left\{X_{n}\right\}$ with spectral density $g$. For $j \geq 1$ we have $\int_{-\pi}^{\pi} \log ^{2}|t-s| g(t) d t \geq \beta_{j} \int_{-\pi}^{\pi} \log ^{2}|t-s| g_{j}(t) d t$. Hence, for every $j \geq 1$ and for every $s \in P_{j}$, we have $\int_{-\pi}^{\pi} \log ^{2}|t-s| g(t) d t=\infty$. We conclude that for every $s \in P$ the series $\sum_{n=1}^{\infty} \frac{\mathrm{e}^{i n s} X_{n}}{n}$ does not converge in norm.

Since each set $P_{j}$ is perfect, so is the closure of $P$. Furthermore, the logarithmic Hausdorff dimension of $P$ is not less than the dimension of any $P_{j}$. So, the logarithmic dimension of $P$ is $\geq 2$. Using Theorem 1.5 we obtain that the logarithmic dimension of $P$ is 2 .

\section{Appendix: Hausdorff dimension of a set of divergence}

\section{1. $h$-Hausdorff dimension.}

We prove in this appendix the result used in Theorem 1.5 (Theorem 3.2). It is likely to belong to the "folklore" of Hausdorff dimension theory, but for the sake of completeness we prove the result which fits exactly to our need. First we recall general notions and results on the construction of Hausdorff measures on a metric space $(X, d)$. For our purpose it suffices to take $X=\mathbb{R}$.

Let $\mathcal{F}$ be a family of subsets of $X$ and $\zeta$ be a map from $\mathcal{F}$ to $[0, \infty]$. A generalized Hausdorff measure $\phi$ on $(X, d)$ is associated to $(\mathcal{F}, \zeta)$ in a standard way: For every $\delta>0$, we define the set function $\phi_{\delta}$ by

$$
\phi_{\delta}(A)=\inf \left\{\sum_{i \in \mathbb{N}} \zeta\left(U_{i}\right)\right\}, A \subset X,
$$

where the infimum is taken over all countable families $\left(U_{i}\right)_{i \in \mathbb{N}}$ of elements of $\mathcal{F}$ such that $A \subset \cup_{i \in \mathbb{N}} U_{i}$ and $\operatorname{diam} U_{i} \leq \delta$ for all $i$. The Hausdorff measure of a subset $A$ is $\phi(A)=\lim _{\delta \rightarrow 0} \phi_{\delta}(A)=\sup _{\delta>0} \phi_{\delta}(A)$.

We will consider functions $\zeta$ of the following form: let $h:[0, \infty[\rightarrow[0, \infty[$ be an increasing continuous function with $h(0)=0$ and let $\mathcal{F}$ be the family all bounded subsets of $\mathbb{R}$. For 
ON THE CONVERGENCE OF THE ROTATED ONE-SIDED ERGODIC HILBERT TRANSFORM 13

each $s>0$, we can take as $\zeta$ the map

$$
\zeta_{s}: \mathcal{F} \rightarrow\left[0, \infty\left[, A \rightarrow h^{s}(\operatorname{diam} A)\right.\right.
$$

The corresponding set functions are denoted by $\phi_{\delta}^{s}$ and $\phi^{s}$.

Using the continuity at 0 and the monotonicity of $h$, it is easy to see that if $\phi^{s}(A)<\infty$ for some $s>0$, then $\phi^{t}(A)=0$ for all $t>s$. The generalized $h$-Hausdorff dimension of $A$ is defined by

$$
\operatorname{dim}_{h}(A):=\inf \left\{s>0: \phi^{s}(A)=0\right\}
$$

We will use the following result on approximation by compact sets (which holds for any Suslin subset, cf. [Fe], p. 186, Corollary 2.10.23).

Theorem 3.1. Let $X$ be a metric space such that all bounded closed subsets are compact and $\mathcal{F}$ be the family of all compact subsets of $X$. Suppose that the map $\zeta$ is continuous for the Hausdorff distance and that $\zeta(C)>0$ whenever $\operatorname{diam} C>0$. Then every Borel subset $S$ of $X$ satisfies

$$
\phi(S)=\sup \{\phi(C): C \in \mathcal{F}, C \subset S\}
$$

Let $G: X \times X \rightarrow[0, \infty]$ be a LSC (lower semi-continuous) kernel. For a positive measure $\mu$ on $X$, for $x \in X$ the potential associated with $\mu$ and $G$, and the potential associated with $\mu$ and the dual kernel $G^{*}(x, y)=G(y, x)$ are defined by

$$
G \mu(x):=\int G(x, y) \mu(d y), G^{*} \mu(x):=\int G(y, x) \mu(d y) .
$$

Since $G$ is LSC, Fatou's lemma implies that $G \mu$ and $G^{*} \mu$ are also LSC. The energy of $\mu$ for the kernel $G$ is

$$
I_{G}(\mu):=\iint G(x, y) \mu(d x) \mu(d y)=\int G \mu(x) \mu(d x)=\int G^{*} \mu(y) \mu(d y) .
$$

\section{2. $h$-Hausdorff dimension of the set of divergence of the potential.}

We are interested by the size of the set where the potential of a measure $\mu$ is infinite for the kernels $G_{s}, s>0$, defined (with the convention $\frac{1}{0}=+\infty$ ) by

$$
G_{s}:(x, y) \in \mathbb{R} \times \mathbb{R} \rightarrow \frac{1}{h^{s}(|x-y|)} .
$$

For a positive measure $\mu$ on $\mathbb{R}$ with finite mass and a parameter $s_{0}>0$, we consider the following set

$$
F_{\mu, s_{0}}:=\left\{x \in \mathbb{R}: G_{s_{0}} \mu(x)=\int_{\mathbb{R}} \frac{1}{h^{s_{0}}(|x-y|)} \mu(d y)=+\infty\right\} .
$$

Theorem 3.2. Let $h:[0,+\infty[\rightarrow[0,+\infty[$ be a continuous increasing function with $h(0)=0$, continuously differentiable outside a discrete subset of $[0,+\infty[$. Assume that there exists a constant $C$ such that $h(2 x) \leq C h(x), \forall x \geq 0$. Then for any $\mu$ with finite mass and $s_{0}>0$, we have $\operatorname{dim}_{h}\left(F_{\mu, s_{0}}\right) \leq s_{0}$. 
The proof is based on the proposition and the lemma below. Some details are straightforward extensions of proofs which can be found in standard books on Hausdorff measures ([9], [10], [4]) and are omitted.

Proposition 3.3. Let $G$ be a LSC kernel such that, for some positive constant $C_{1}$,

$$
\forall x, y, z \in X, d(y, z) \leq 3 d(x, z) \Rightarrow G(z, x) \leq C_{1} G(z, y) .
$$

Let $\mu$ be a finite positive measure on $X$ and let $F=\{x \in X: G \mu(x)=+\infty\}$. Then the energy $I_{G}(\nu)$ of any non zero positive measure $\nu$ with support in $F$ is infinite.

Proof. Let $\lambda$ be a positive measure and $E=\operatorname{supp} \lambda$. For all $x \in X$, there exists $y \in E$ such that $d(x, y) \leq 2 d(x, E)$. For all $z \in E$, we have $d(y, x) \leq 2 d(x, E) \leq 2 d(x, z)$, therefore $d(y, z) \leq d(y, x)+d(x, z) \leq 3 d(x, z)$. Hence $G(z, x) \leq C_{1} G(z, y)$ and

$$
G^{*} \lambda(x)=\int G(z, x) \lambda(d z) \leq \int C_{1} G(z, y) \lambda(d z)=C_{1} G^{*} \lambda(y) .
$$

Therefore

$$
\sup _{x \in X} G^{*} \lambda(x) \leq C_{1} \sup _{y \in E} G^{*} \lambda(y) .
$$

Moreover suppose that $E=\operatorname{supp} \lambda \subset \mathrm{F}$, then

$$
+\infty=\int G \mu(y) \lambda(d y)=\int G^{*} \lambda(x) \mu(d x) .
$$

Since $\mu$ is finite, it follows that $\sup _{x \in X} G^{*} \lambda(x)=+\infty$. Hence $\sup _{y \in E} G^{*} \lambda(y)=+\infty$.

Now let $\nu$ be a non zero positive measure positive with $\operatorname{supp} \nu \subset \mathrm{F}$, and assume that the energy of $\nu$ is finite, $\int G^{*} \nu(x) \nu(d x)=I_{G}(\nu)<+\infty$. We will apply the first part of the proof to a measure $\lambda$ deduced from $\nu$ and get a contradiction.

For all $a>0$, we have $\nu\left(\left\{x \in X: G^{*} \nu(x)>a\right\}\right) \leq I_{G}(\nu) / a$. Hence, if $a$ is large enough, the set $A=\left\{x \in X: G^{*} \nu(x) \leq a\right\}$ has a positive measure. Choose such a number $a$. The set $A$ is closed, since $G^{*} \nu$ is LSC.

Consider the measure $\lambda$ defined by $\lambda(B)=\nu(A \cap B)$ for all Borel subsets $B$ of $X$. On the one hand, the choice of $a$ ensures that $\lambda$ is not zero. On the other hand, $\lambda \leq \nu$ and therefore $G^{*} \lambda \leq G^{*} \nu \leq a$ on the set $A$ since $A$ contains $E=\operatorname{supp} \lambda$. This contradicts $\sup _{y \in E} G^{*} \lambda(y)=+\infty$, and therefore the energy of $\nu$ cannot be finite.

Lemma 3.4. If $\phi^{s}\left(F_{\mu, s_{0}}\right)>0$ for some $s>0$, there exists a finite positive measure $\nu_{0}$ with support in $F_{\mu, s_{0}}$ such that, for all $0<t<s$,

$$
I_{G_{t}}\left(\nu_{0}\right)=\iint \frac{1}{h^{t}(|x-y|)} \nu_{0}(d x) \nu_{0}(d y)<+\infty .
$$

Proof. The measure $\nu_{0}$ is constructed by restricting $\phi^{s}$ to a suitable compact subset $K_{0}$ of $F_{\mu, s_{0}}$.

1) The continuity of the kernel $G_{s}:(x, y) \in \mathbb{R} \times \mathbb{R} \rightarrow \frac{1}{h^{s}(|x-y|)} \in[0,+\infty]$ implies that $F_{\mu, s_{0}}$ is a $G_{\delta}$ and hence a Borel subset of $\mathbb{R}$. Theorem 3.1 with $S=F_{\mu, s_{0}}$ and $\phi=\phi^{s}$ provides a compact set $K_{1} \subset F_{\mu, s_{0}}$ such that $\phi^{s}\left(K_{1}\right)>0$. 
Now there is a compact subset $K_{2} \subset K_{1}$ such that $0<\phi^{s}\left(K_{2}\right)<+\infty$. The proof of this assertion can be easily adapted from [9] p. 62, Theorem 4.10, where the same result is proved for the usual Hausdorff measure.

2) Using the inequality $h(2 x) \leq C h(x)$ and standard arguments (covering lemma and Egoroff's theorem (cf. [9] Proposition 4.9 p. 61 and Proposition 4.11 p. 63)) one can show that there exists a compact subset $K_{0} \subset K_{2}$ and a finite constant $b$ such that $\phi^{s}\left(K_{0}\right)>0$ and

$$
\phi^{s}\left(K_{0} \cap B(x, r)\right) \leq b h^{s}(r), \forall x \in \mathbb{R}, r>0 .
$$

Let $\nu_{0}$ be the measure defined by $\nu_{0}(A)=\phi^{s}\left(K_{0} \cap A\right)$. For $0<t<s$, let $G_{t} \nu_{0}(x):=$ $\int_{\mathbb{R}} \frac{\nu_{0}(d y)}{h^{t}(|x-y|)}$. For all $x \in \mathbb{R}$ and $r \geq 0$, set $m_{x}(r)=\nu_{0}(B(x, r))$. We have $m_{x}(0)=0$ since $h^{s}(r)$ tends to 0 as $r \rightarrow 0$, and $m_{x}(r) \leq b h^{s}(r)$ by (21). By the same computation as in [9, p. 65-66], we obtain:

$$
\begin{aligned}
& G_{t} \nu_{0}(x)=\int_{B(x, 1)} \frac{\nu_{0}(d y)}{h^{t}(|x-y|)}+\int_{B(x, 1)^{C}} \frac{\nu_{0}(d y)}{h^{t}(|x-y|)} \leq \\
& \int_{0}^{1} h^{-t}(r) d m_{x}(r)+\nu_{0}(\mathbb{R}) h^{-t}(1) \\
& =\left[h^{-t}(r) m_{x}(r)\right]_{0}^{1}+\int_{0}^{1} t h^{-t-1}(r) h^{\prime}(r) m_{x}(r) d r+\nu_{0}(\mathbb{R}) h^{-t}(1) \\
& \leq h^{-t}(1) b h^{s}(1)+\int_{0}^{1} t h^{-t-1}(r) h^{\prime}(r) b h^{s}(r) d r+\nu_{0}(\mathbb{R}) h^{-t}(1) \\
& \leq b+\nu_{0}(\mathbb{R}) h^{-t}(1)+b t\left[\frac{h^{s-t}(r)}{s-t}\right]_{0}^{1} .
\end{aligned}
$$

Therefore, $G_{t} \nu_{0}$ is bounded on $\mathbb{R}$ and $I_{G_{t}}\left(\nu_{0}\right)<+\infty$.

Proof of Theorem 3.2 Let $\mu$ be a positive finite measure on $\mathbb{R}$ and let $s_{0}>0$. Suppose that there exists $s>s_{0}$ such that $\phi^{s}\left(F_{\mu, s_{0}}\right)>0$, where $F_{\mu, s_{0}}$ is defined by (20). It follows from Proposition 3.3 that for all positive measures $\nu$ with support in $F_{\mu, s_{0}}$, $I_{G_{s_{0}}}(\nu)=+\infty$. But this contradicts the existence of a measure $\nu_{0}$ with support in $F_{\mu, s_{0}}$, such that $I_{G_{s_{0}}}(\nu)<+\infty$, as asserted by Lemma 3.4.

Acknowledgements: Parts of this research were carried out during a visit of the second author to the University of Rennes 1 and during a visit of the third author to the Center for Advanced Studies in Mathematics at Ben Gurion University. These authors are grateful to their hosts for their hospitality and support. The authors would like to thank Christophe Cuny and Michael Lin for their interest in this paper, and in particular Christophe Cuny for his suggestions related to Section 2. 


\section{REFERENCES}

[1] I. Assani, Spectral characterization of Wiener-Wintner dynamical systems, Ergod. Th. \& Dynam. Sys. 24 (2004), 347-365.

[2] I. Assani and M. Lin, On the one-sided ergodic Hilbert transform, Contemp. Math. 430 (2007), 221-39.

[3] J. Campbell, Spectral analysis of the ergodic Hilbert transform, Indiana Univ. Math. J. 35 (1986), 379-390.

[4] L. Carleson, Selected problems on exceptional sets. Van Nostrand Mathematical Studies, No. 13 D. Van Nostrand 1967.

[5] G. Cohen and M. Lin, The one-sided ergodic Hilbert transform of normal contractions, in Characteristic Functions, Scattering Functions and Transfer Functions. Birkhäuser, Basel 2009, 77-98.

[6] C. Cuny, On the a.s. convergence of the one-sided ergodic Hilbert transform, Ergod. Th. E Dynam. Sys. 29 (2009), 1781-1788.

[7] C. Cuny, Pointwise ergodic theorems with rate and application to limit theorem for stationary processes, preprint (see arXiv:0904.0185v1).

[8] J. L. Doob, Stochastic Processes. John Wiley \& sons, New York 1953.

[9] K. Falconer, Fractal geometry. Mathematical foundations and applications. John Wiley \& Sons, Ltd., Chichester, 1990. xxii+288 pp..

[10] H. Federer, Geometric measure theory. Die Grundlehren der mathematischen Wissenschaften, Band 153 Springer-Verlag New York Inc., New York 1969 xiv+676 pp.

[11] V. Gaposhkin, Spectral criteria for existence of generalized ergodic transforms, Theory of probability and its appl. 41 (1996), 247-264.

[12] A. del Junco and J. Rosenblatt, Counterexamples in ergodic theory and number theory, Math. Ann. 245 (1979), no. 3, 185-197.

[13] J.P. Kahane, Some random series of functions, 2nd ed., Cambridge University Press, 1985.

[14] M. Lacey and E. Terwilleger, A Wiener-Wintner theorem for the Hilbert transform, Ark. Mat. 46 (2008), no. 2, 315-336.

[15] F. Riesz and B. Sz. Nagy, Functional Analysis. Translated from the $2^{\text {nd }}$ French edition by Leo F. Boron, Dover Publications Inc., New York 1990.

[16] N. Wiener and A. Wintner, Harmonic analysis and ergodic theory, Amer. J. Math. 63 (1941), 415-426.

[17] A. Zygmund, Trigonometric series, vol I, II, corrected 2nd ed., Cambridge University Press, Cambridge, 1968.

Nicolas Chevallier, Faculté des Sciences et Techniques, Université de Haute Alsace, 68093 Mulhouse, France

E-mail address: nicolas.chevallier@uha.fr

Guy Cohen, Dept. of Electrical Engineering, Ben-Gurion University, Israel

E-mail address: guycohen@ee.bgu.ac.il

Jean-Pierre Conze, IRMAR, CNRS UMR 6625, University of Rennes I, Campus de Beaulieu, 35042 Rennes Cedex, France

E-mail address: conze@univ-rennes1.fr 